\title{
EXPANSIONS OF DISTRIBUTIONS IN TERMS OF GENERALIZED HEAT POLYNOMIALS AND THEIR APPELL TRANSFORMS
}

\author{
R. S. PATHAK \\ Department of Mathematics, Banaras Hindu University \\ Varanasi, India \\ and \\ LOKENATH DEBNATH \\ Mathematical Institute, University of Oxford \\ Oxford, England \\ (Received November 13, 1978 and in revised form July 15, 1980)
}

ABSTRACT. This paper is concerned with expansions of distributions in terms of the generalized heat polynomials and of their Appell transforms. Two different techniques are used to prove theorems concerning expansions of distributions. A theorem which provides an orthogonal series expansion of generalized functions is also established. It is shown that this theorem gives an inversion formula for a certain generalized integral transformation.

KEY WORDS AND PHRASES. Expansion of distributions (or generalized functions), Generalized heat polynomials and their Appell transforms, Orthogonal Series expansion of generalized functions and generalized integral transformation.

1980 MATHEMATICS SUBJECT CLASSIFICATION CODES. $46 F 12,46 F 10,44 A 05$.

1. INTRODUCTION.

Zemanian [1] has developed a procedure for expanding certain Schwartz distributions (or generalized functions) in a series of the Fourier type. He has also shown that every distribution of polynomial growth (or tempered distribution) can be expanded in a series of Hermite functions. Some general results concerning the orthogonal series expansion of generalized functions are also available in recent literature including reference listed by Zemanian [1].

In a paper, Korevaar [2] has developed a general theory of Fourier transforms 
and pansions based upon the ideas different from those of the Schwartz-Sobolev approach [3], and from those of Ehrenpreis [4]. This theory did not include the notion of convergence for pansions as there exists such notions for distributions. In fact, the theory is essentially in algebraic in nature. It is also shown that distributions which are finite order derivatives of certain functions growing no faster than $\exp \left(\alpha x^{2}\right), \alpha<\frac{1}{2}$ are uniquely determined by their Hermite series. However, no topology was introduced there, and hence there is no way of expressing the generalized function as a functional in terms of the Hermite coefficients.

In two papers [5-6], Haimo studied the expansion of functions in terms of heat polynomials and their Appell transforms. This work is an extension of some results of Rosenbloom and Widder [7] on expansions in terms of heat polynomials and associated functions. Despite these works, no attention is given to the expansions of distributions in terms of heat polynomials and their Appell transforms.

This paper is devoted to the study of expansions of distributions in terms of the generalized heat polynomials and their Appell transforms. A theorem which provides an orthogonal series expansion of generalized functions is also proved. It is shown that this theorem gives an inversion formula for a certain generalized integral transformation.

\section{PRELIMINARY RESULTS ON THE GENERALIZED HEAT POLYNOMIALS.}

For real values of $x$ and $t$, the generalized heat polynomials, $P_{n, v}(x, t)$ and its Appell transform, $w_{n, v}(x, t)$ are defined by

$$
\begin{aligned}
& p_{n, \nu}(x, t)=\sum_{r=0}^{n} 2^{2 r}\left(\begin{array}{l}
n \\
r
\end{array}\right) \frac{\Gamma\left(\nu+\frac{1}{2}\right)}{\Gamma\left(\nu+\frac{1}{2}+n-r\right)} x^{2(n-r)} t^{r}, \\
& w_{n, \nu}(x, t)=t^{-2 n} G(x, t) p_{n, \nu}(x,-t), \quad t>0,
\end{aligned}
$$

where $n=0,1,2, \ldots ; \nu$ is a fixed positive number and $G(x, t)$ is given by

$$
G(x, t)=(2 t)^{-\left(\nu+\frac{1}{2}\right)} \exp \left(-\frac{x^{2}}{4 t}\right) \text {. }
$$

When $\nu=0$, it follows from (2.1) that

$$
\begin{aligned}
& P_{n, o}(x, t)=v_{2 n}(x, t), \\
& P_{n, o}(x,-1)=H_{2 n}\left(\frac{x}{2}\right),
\end{aligned}
$$


where $v_{2 n}(x, t)$ is the ordinary heat polynomial of even order defined by Rosenbloom and Widder [7, p. 222], and $\mathrm{H}_{2 \mathrm{n}}$ is the Hermite polynomial of even order given in Erdélyi's book [8].

It can readily be verified that for $-\infty<x, t<\infty, P_{n, v}(x, t)$ satifies the generalized heat equation

$$
\Delta_{x} u(x, t)=\frac{\partial u}{\partial t}
$$

where the operator $\Delta_{x}=\frac{\partial^{2}}{\partial x^{2}}+\left(\frac{2 \nu}{x}\right) \frac{\partial}{\partial x}$ with some fixed positive number $\nu$.

It is noted that $W_{n, v}(x, t)$ is also a solution of $(2.6)$. Further, $W_{n, v}(x, t)$ satifies the following operator formulas

$$
W_{n, \nu}(x, t)=2^{2 n} \Delta_{x}^{n} G(x, t),
$$

with

$$
\Delta_{x}^{k} W_{n, v}(x, t)=2^{-2 k} w_{n+k, v}(x, t)
$$

With the help of the following results reported by Erdélyi [8]

$$
\begin{aligned}
P_{n, v}(x,-t)= & (-1)^{n} 2^{2 n} n ! t^{n} L_{n}^{\left(\nu-\frac{1}{2}\right)}\left(\frac{x^{2}}{4 t}\right), t>0, \\
& \left|L_{n}^{\alpha}(x)\right| \leq \Delta_{n, \alpha} \exp \left(\frac{x}{2}\right),
\end{aligned}
$$

where $L_{n}^{\alpha}(x)$ is the Laguerre polynomial of degree $n$ and order $\alpha>-1$ and

$$
\Delta_{\mathrm{n}, \alpha}=\left[\begin{array}{lr}
\frac{1}{\mathrm{n} !}(\alpha+1)_{\mathrm{n}}, & \alpha \geq 0 \\
2-\frac{1}{\mathrm{n} !}(\alpha+1)_{\mathrm{n}}, & -1<\alpha<0
\end{array}\right],
$$

with $(\alpha)_{n}=\frac{\Gamma(n+\alpha)}{\Gamma(\alpha)}$; it can easily be shown that for $t>0$

$$
\begin{gathered}
\left|P_{n, v}(x,-t)\right| \leq n: 2^{2 n} \Delta_{n, v-\frac{1}{2}} t^{n} \exp \left(\frac{x^{2}}{8 t}\right), \\
\left|W_{n, v}(x, t)\right| \leq 2^{2 n-v-\frac{1}{2}} n ! \Delta_{n, v-\frac{1}{2}} t^{-\left(n+v+\frac{1}{2}\right)} \exp \left(-\frac{x^{2}}{8 t}\right) .
\end{gathered}
$$

The differential $\mathrm{d} \mu(\mathrm{x})$ is defined in a paper of Haimo [6] as

$$
\mathrm{d} \mu(\mathrm{x})=2^{\left(\frac{1}{2}-\nu\right)}\left[\Gamma\left(\nu+\frac{1}{2}\right)\right]^{-1} \mathrm{x}^{2 \nu} \mathrm{dx}
$$

It is interesting to observe that $P_{n, v}(x, t)$ form a biorthogonal system in $0<\mathbf{x}<\infty$. Indeed, 


$$
\int_{0}^{\infty} W_{n, \nu}(x, t) P_{m, \nu}(x,-t) d \mu(x)=\frac{\delta_{n m}}{b_{n}} t>0,
$$

where

$$
b_{n}=\Gamma\left(\nu+\frac{1}{2}\right) /\left[2^{4 n} n ! \Gamma\left(\nu+n+\frac{1}{2}\right)\right] .
$$

An important consequence of $(2.15)-(2.16)$ is the bilinear generating function for the biorthogonal set $P_{n, \nu}$ and $W_{n, \nu}$ as

$$
G(x, y ; s+t)=\sum_{n=0}^{\infty} b_{n} w_{n, v}(y, s) P_{n, v}(x, t) .
$$

The source solution of $(2.6)$ is given by $G(x, t)$ in the form

$$
\begin{gathered}
G(x, y ; t)=(2 t)^{-\left(\nu+\frac{1}{2}\right)} \exp \left[-\left(x^{2}+y^{2}\right) / 4 t\right] d\left(\frac{x y}{2 t}\right), \\
d(x)=2^{\nu-\frac{1}{2}} \Gamma\left(\nu+\frac{1}{2}\right) x^{\frac{1}{2}-\nu} I_{\nu-\frac{1}{2}}(x),
\end{gathered}
$$

and $I_{r}(x)$ is the modified Bessel function of imaginary argument of order $r$; and $G(x, 0 ; t)=G(x, t)$.

\section{THE TEST FUNCTION SPACE $U_{\sigma, \nu^{\circ}}$}

We denote the open interval $(0, \infty)$ by $I$. A complex valued infinitely differentiable function $\phi(x)$ defined over I belongs to the space $U_{\sigma, \nu}(I)$ if

$$
\gamma_{k}(\phi) \triangleq \sup _{0<x<\infty}\left|\exp \left(\frac{x^{2}}{4 \sigma}\right) \Delta_{x}^{k} \phi(x)\right|<\infty,
$$

for any fixed $k$, where $k$ assumes the values $0,1,2, \ldots ; \sigma$ is a positive real number, $\nu>-\frac{1}{2}$ and $\Delta_{x}$ is the differential operator involved in section 2 .

The topology in the space $U_{\sigma, \nu}$ is induced by the collection of seminorms $\left\{\gamma_{k}\right\}_{k=0}^{k=\infty}$. Since $\gamma_{0}$ is a norm, the collection of seminorms is separating as indicated by Zemanian $[1, p 8]$. A sequence $\left\{\phi_{r}\right\}_{1}^{\infty}$ is said to converge to $\phi$ in $U_{\sigma, v}$ (I) if for each $k, \gamma_{k}\left(\phi_{r}-\phi\right)$ tends to zero as $r \rightarrow \infty$. A sequence $\left\{\phi_{r}\right\}_{r=1}^{\infty}$ with each $\phi_{r}(x)$ belonging to $U_{\sigma, v}(I)$ is a Cauchy sequence in $U_{\sigma, v}(I)$ if $\gamma_{k}\left(\phi_{r}-\phi_{s}\right) \rightarrow 0$ as $r, s \rightarrow \infty$ independently of each other for every fixed $k$, where $k=0,1,2, \ldots$. It is noted here that $U_{\sigma, \nu}(I)$ is a locally convex, sequentially complete, Hausdorff topological linear space. Its dual space $U_{\sigma, \nu}^{\prime}(I)$ is the space of generalized functions under consideration. 
From the estimate $(2.10)$, it follows that for $x>0, s>\frac{\sigma}{2}$ and $v>-\frac{1}{2}$, $\mu(x) P_{n, \nu}(x,-s) \in U_{\sigma, \nu}(I)$. Using results $(2.4),(2.5)$ and $(2.8)$, it can also be seen that $W_{n, v}(x, t) \in U_{\sigma, v}(I)$ for $x>0$ and $0<t<\sigma$.

In order to make this paper self-contained to some extent, we now 1 ist a few properties of the above spaces:

(i) $\mathcal{O}(I)$ denotes the space of infinitely differentiable functions defined over I with a compact support. The dual space $D^{\prime}(I)$ is the space of Schwartz distributions [9] on I. It can easily be shown that $D(I)$ is a subspace of the space $U_{\sigma, \nu}$ (I) and that the topology of $D(I)$ is stronger than that induced on it by $U_{\sigma, \nu}(I)$. (ii) The space $U_{\sigma, \nu}(I)$ is a dense subspace of $\varepsilon(I)$ which is the space of all complex-valued smooth functions on $I$. The topology of $U_{\sigma, \nu}$ is stronger than the topology induced on $U_{\sigma, \nu}$ by $\mathcal{E}(I)$. So $\mathcal{E}^{\prime}(I)$ can be identified with a subspace of $U_{\sigma, \nu}^{\prime}$

(iii) The space $S^{\prime}$ of tempered distributions is a subspace of $U_{\sigma, \nu}^{\prime}$ for $\nu>\frac{1}{2}$ [Pandey, 10].

(iv) For each $f \in U_{\sigma, \nu}^{\prime}$ there exists a non-negative integer $r$ and a positive constant $\mathrm{C}$ such that

$$
|\langle\mathrm{f}, \phi\rangle| \leq \mathrm{C} \max _{0 \leq \mathrm{k} \leq \mathrm{r}} \gamma_{\mathrm{k}}(\phi),
$$

for every $\phi \in U_{\sigma, \nu}$, where $r$ and $C$ depend on $f$ but not on $\phi[8, p 19]$. (v) If $f(x)$ is a locally integrable for $x>0$ such that $f(x) \exp \left(-\frac{x^{2}}{4 \sigma}\right)$ is absolutely integrable on $0<x<\infty$, then $f(x)$ generates a regular generalized function $f \in U_{\sigma, \nu}^{\prime}$ defined by the integral

$$
\langle\mathrm{f}, \phi\rangle=\int_{0}^{\infty} \mathrm{f}(\mathrm{x}) \overline{\phi(\mathrm{x})} \mathrm{dx},
$$

where $\quad \phi(x) \in U_{\sigma, \nu}(I)$.

(vi) Let $f(x)$ be a locally integrable function defined for $x>0$ such that

$$
f(x)=\left[\begin{array}{lll}
0\left(\mathrm{x}^{\rho}\right) & \rho+1>0, & \mathrm{x} \rightarrow 0+ \\
0\left(\mathrm{e}^{\delta \mathrm{x}^{2}}\right) & 0<\delta<1 /(4 \sigma), x \rightarrow \infty
\end{array}\right]
$$

Then, clearly $f \in U_{\sigma, \nu}^{\prime}$. Let A denote Zemanian's test function space ([1], p252). 
Then $\phi(x)=x^{\frac{1}{2} \alpha} e^{-x / 2} L_{n}{ }^{(\alpha)}(x) \in A$. But $\int_{0}^{\infty} f(x) \overline{\phi(x)}$ dx does not exist. Hence, $f(x)$ satisfying (2.4) does not belong to $A^{\prime}([1], p 258)$. Therefore, $U_{\sigma, \nu}^{\prime} \not A^{\prime}$.

Since $D(I)$ is contained in both the spaces $U_{\sigma, \nu}$ and $A$, it follows that the spaces $U_{\sigma, \nu}^{\prime}$ and $A^{\prime}$ over-1ap but $U_{\sigma, \nu}^{\prime} \not A^{\prime}$.

\section{EXPANSION OF DISTRIBUTIONS.}

The expansion of $f \in U_{\sigma, \nu}^{\prime}$ in terms of heat polynomials $P_{n, \nu}(x,-t)$ and their Appell transforms $\mathrm{w}_{\mathrm{n}, \nu}(\mathrm{x}, \mathrm{t})$ is given in the following theorem:

THEOREM 4.1. Let $\mathrm{f} \in \mathrm{U}_{\sigma, \nu}^{\prime}$ where $\nu>\frac{1}{2}$ and $\sigma>0$. Then, for $0<\sigma / 2<\mathrm{t}<\sigma$,

$$
\langle f(x), \phi(x)\rangle=\lim _{s \rightarrow t-} \lim _{N \rightarrow \infty} \sum_{n=0}^{N} k_{n} a_{n}(t)\left\langle\mu(x) P_{n, \nu}(x,-s), \phi(x)\right\rangle,(4.1)
$$

where

$$
\begin{aligned}
& a_{n}(t) \triangleq\left\langle f(y), \quad w_{n, \nu}(y, t)\right\rangle, \\
& K_{n}=\Gamma\left(\nu+\frac{1}{2}\right) /\left[2^{4 n} n: \Gamma\left(\nu+\frac{1}{2}+n\right)\right] .
\end{aligned}
$$

We shall need the following lammas for the proof of this theorem.

LEMMA 4.1. For $\mathrm{f} \in \mathrm{U}_{\sigma, \nu}^{\prime}$ where $\nu>0$ and $\sigma>0$, we define

$$
a_{n}(t) \triangleq\left\langle f(y), w_{n, \nu}(y, t)\right\rangle, \quad n=0,1,2, \ldots,
$$

Then for $0<t<\sigma$,

$$
\left|a_{n}(t)\right| \leq C(4 / t)^{n}\left[\left(\nu+\frac{1}{2}\right)_{n+r}+2(n+r) !\right],
$$

where $\mathrm{C}$ and $\mathrm{r}$ are independent of $\mathrm{n}$.

PROOF. By the boundedness property of generalized functions there exist a positive constant $C$ and a non-negative integer $r$ such that

$$
\begin{aligned}
\left|a_{n}\right| & =\left|\left\langle f(y), w_{n, \nu}(y, t)\right\rangle\right| \\
& \leq C \max _{0 \leq k \leq r} \gamma_{k}\left(w_{n, \nu}(y, t)\right) \\
& =C \max _{0} \sup _{0<\mathrm{k}} \leq r \quad \mid 2^{-2 k} e^{y^{2 /(4 \sigma)} W_{n+k, v}(y, t) \mid .}
\end{aligned}
$$

We arrive at the desired estimate using result (2.11).

LEMMA 4.2. Let $\phi(x) \in U_{\sigma, \nu}$. Then, for $0<\sigma / 2<s<t<\sigma$,

$$
\exp \left(y^{2} / 4 \sigma\right)\left|\Delta_{y}^{k} \int_{0}^{\infty} \sum_{n=N}^{\infty} k_{n} w_{n, \nu}(y, t) P_{n, \nu}(x,-s) \phi(x) d \mu(x)\right| \rightarrow 0
$$

as $\mathrm{N} \rightarrow \infty$ uniformly for all $\mathrm{y}>0$. 
PROOF. Using the estimates $(2.10)$ and (2.11) we can write

$$
\begin{aligned}
|J| & \equiv \exp \left(y^{2} / 4 \sigma\right)\left|\Delta_{y}^{k} \int_{0}^{\infty} \sum_{n=N}^{\infty} k_{n} w_{n, \nu}(y, t) P_{n, \nu}(x,-s) \phi(x) d \mu(x)\right| \\
& \leq \exp \left(y^{2} / 4 \sigma\right) \int_{0}^{\infty} \sum_{n=N}^{\infty} k_{n} \frac{2^{\frac{1}{2}-\nu-2 k}}{\Gamma\left(\nu+\frac{1}{2}\right)}\left|w_{n+k, \nu}(y, t)\right|\left|P_{n, \nu}(x,-s)\right||\phi(x)| d \mu(x) \\
& \leq \exp \left[-\frac{y^{2}}{4 \sigma}\left(\frac{1}{t}-\frac{1}{\sigma}\right)\right] \sum_{n=N}^{\infty} \frac{\Gamma\left(\nu+\frac{1}{2}\right)}{\Gamma\left(\nu+\frac{1}{2}+n\right)}(n+k) ! \Delta_{n+k, \nu-\frac{1}{2}} \Delta_{n, \nu-\frac{1}{2}} 2^{2 k-v-\frac{1}{2}} x \\
& x t^{-2 k-\nu-\frac{1}{2}}(s / t)^{n} \int_{0}^{\infty} \exp \left(x^{2} / 8 s\right)|\phi(x)| d \mu(x) .
\end{aligned}
$$

Since $\phi(x) \in U_{\sigma, \nu}$, there exists a positive constant $C$ such that

$$
\begin{aligned}
|J| & \leq c \sum_{n=N}^{\infty} \frac{\Gamma(\nu+1+k+n)}{(n+1)}(s / t)^{n} \\
& \leq C_{1} \sum_{n=N}^{\infty} n^{v+k-\frac{1}{2}}(s / t)^{n},
\end{aligned}
$$

where $C_{1}$ is another positive constant. Clearly the last term tends to zero as $N \rightarrow \infty$ for $s<t$.

LEMMA 4.3. For $0<\sigma / 2<s<t<\sigma$, let $G(x, y ; t-s)$ be the function defined by (2.14) and let $\phi(x) \in U_{\sigma, \nu} \cdot$ Then there exist $\varepsilon>0$ and a large positive constant $A_{1}(n)$ such that for $y>A_{1}(n)$,

$$
\exp \left(\mathrm{y}^{2} / 4 \sigma\right)\left|\int_{0}^{\infty} \Delta_{\mathrm{y}}^{\mathrm{k}}\{G(\mathrm{x}, \mathrm{y}, \mathrm{t}-\mathrm{s})\} \quad \phi(\mathrm{x}) \mathrm{d} \mu(\mathrm{x})\right|<\frac{\varepsilon}{2}, \quad \mathrm{k}=0,1,2, \ldots .
$$

PROOF. Proceeding as in the proof of Lemma 4.2, we write

$$
\begin{aligned}
& \exp \left(\mathrm{y}^{2} / 4 \sigma\right)\left|\int_{0}^{\infty} \Delta_{\mathrm{y}}^{\mathrm{k}}\{\mathrm{G}(\mathrm{x}, \mathrm{y}, \mathrm{t}-\mathrm{s})\} \phi(\mathrm{x}) \mathrm{d} \mu(\mathrm{x})\right| \\
& \leq \mathrm{C}_{2} \exp \left[-\mathrm{y}^{2} / 4\left(\frac{1}{\mathrm{t}}-\frac{1}{\sigma}\right)\right] \sum_{\mathrm{n}=0}^{\infty} \frac{\Gamma\left(\nu+\frac{1}{2}+\mathrm{k}+\mathrm{n}\right)}{\Gamma(\mathrm{n}+1)}\left(\frac{\mathrm{s}}{\mathrm{t}}\right)^{\mathrm{n}} \\
& =\mathrm{C}_{3} \exp \left[-\mathrm{y}^{2} / 4\left(\frac{1}{\mathrm{t}}-\frac{1}{\sigma}\right)\right]\left(1-\frac{\mathrm{s}}{\mathrm{t}}\right)^{-v-\mathrm{k}-\frac{1}{2}}
\end{aligned}
$$

for appropriate constants $\mathrm{C}_{2}$ and $\mathrm{C}_{3}$.

Hence for $t<\sigma$, the last expression tends to zero as $\mathrm{y} \rightarrow \infty$. 
LEMMA 4.4. Let $A_{1}, \varepsilon, G(x, y, t-s)$ and $\phi(x)$ be the same as in Lemma 4.3 and let $\nu>\frac{1}{2}$. Then for $t-s<\delta(n, \varepsilon)$,

$$
\exp \left(y^{2} / 4 \sigma\right)\left|\int_{0}^{\infty} \Delta_{y}^{k}\{G(x, y, t-s)\} \phi(x) d \mu(x)-\phi_{k}(y)\right|<\varepsilon
$$

where $\phi_{k}(y)=\Delta_{y}^{k} \phi(y)$, uniformly for all y $\in\left(0, A_{1}\right)$.

PROOF. If $\phi(x), U_{\sigma, \nu}$, then proceeding as in [10, p846], it follows that for $v>\frac{1}{2}$,

$$
\begin{aligned}
& \phi^{(k)}(x)=0\left(e^{-c x}\right), c>0, \quad x+\infty \\
& \phi^{(k)}(0+) \quad \text { exist finitely, }
\end{aligned}
$$

for each $k=0,1,2, \ldots$. Using these orders of $\phi^{(k)}(x)$ and the identity $\Delta_{x} G(x, y, t)=\Delta_{y} G(x, y, t)$ and integrating by parts, we obtain

$$
\int_{0}^{\infty} \Delta_{y}\{G(x, y, t-s)\} \phi(x) d \mu(x)=\int_{0}^{\infty} G(x, y, t-s) \Delta_{x} \phi(x) d \mu(x) .
$$

Consequently

$$
\int_{0}^{\infty} \Delta_{y}^{k}\{G(x, y, t-s)\} d \mu(x)-\phi_{k}(y)=\int_{0}^{\infty} G(x, y, t-s)\left[\phi_{k}(x)-\phi_{k}(y)\right] d \mu(x),
$$

where we have made use of the fact that

$$
\int_{0}^{\infty} G(x, y, t-s) d \mu(x)=1
$$

We next use the standard technique [10] and remember that $\phi(x)$ is not an element of $D(I)$ although $1 t$ does belong to $U_{\sigma, \nu}$ to show

$$
\exp \left(y^{2} / 4 \sigma\right)\left|\int^{\infty} G(x, y, t-s)\left\{\phi_{k}(x)-\phi_{k}(y)\right\} d \mu(x)\right|<\varepsilon
$$

uniformly for all y satisfying $0<\mathrm{y} \leq \mathrm{A}_{1}$.

We are now prepared to prove the main expansion theorem 4.1 .

PROOF OF THEOREM 4.1. Let $t$ be a fixed number such that $0<\frac{\sigma}{2}<<t<\sigma$ Then using the estimates (2.10) and (4.5), we obtain 


$$
\begin{aligned}
& \sum_{n=0}^{\infty} k_{n}\left|a_{n}\right|\left|\left\langle\mu(x) P_{n, \nu}(x,-s), \phi(x)\right\rangle\right| \\
& \leq C \Gamma\left(\nu+\frac{1}{2}\right) \sum_{n=0}^{\infty} \frac{\left(\nu+\frac{1}{2}\right)_{n+r}+2(n+r) !}{\Gamma\left(\nu+\frac{1}{2}+r\right)} \Delta_{n, \nu-\frac{1}{2}}\left(\frac{s}{t}\right)^{n} \\
& =C \sum_{n=0}^{\infty} \frac{\Gamma\left(\nu+\frac{1}{2}+r+n\right)}{n !}\left(\frac{s}{t}\right)^{n}+2 c \sum_{n=0}^{\infty} \frac{(n+r) !}{n !}\left(\frac{s}{t}\right)^{n}, \quad\left(\nu>\frac{1}{2}\right) \\
& \leq \mathrm{C}_{1} \text {, }
\end{aligned}
$$

where $C_{1}$ is a certain positive constant. This proves the existence of the limit $\mathbf{N} \rightarrow \infty$, when $s<t$.

Using the linearity of $U_{\sigma, \nu}$ and $U_{\sigma, \nu}^{\prime}$ we can write

$$
\begin{aligned}
& \sum_{n=0}^{N} a_{n} k_{n}\left\langle\mu(x) P_{n, v}(x,-s), \phi(x)\right\rangle \\
= & \sum_{n=0}^{N}\left\langle f(y), W_{n, v}(y, t)\right\rangle\left\langle\mu(x) k_{n} P_{n, v}(x,-s), \phi(x)\right\rangle \\
= & \sum_{n=0}^{N}\left\langle f(y), W_{n, v}(y, t)\right\rangle\left\langle\mu(x) k_{n} P_{n, v}(x,-s), \phi(x)\right\rangle \\
= & \left\langle f(y),\left\langle\sum_{n=0}^{N} \mu(x) k_{n} W_{n, v}(y, t) P_{n, v}(x,-s), \phi(x)\right\rangle\right\rangle \\
= & \left\langle f(y),\left\langle\mu(x) G_{N}(x, y, t, s), \phi(x)\right\rangle\right\rangle
\end{aligned}
$$

where

$$
G_{N}(x, y, t, s)=\sum_{n=0}^{N} k_{n} w_{n, v}(y, t) P_{n, v}(x,-s) .
$$

The corresponding infinite series equals $G(x, y, t-s)$. We observe that

$\mu(x) G(x, y, t-s) \in U_{\sigma, \nu}^{\prime}$ and $\mu(x) G_{N}(x, y, t, s), \in U_{\sigma, \nu}^{\prime}$ for $s>\frac{\sigma}{2}$. Furthermore, as a function of $y,\langle\mu(x) G(x, y, t-s), \phi(x)\rangle$ is an element of $U_{\sigma, \nu}$. From Lemma 4.2, we know that

$$
\left\langle\mu(x) G_{N}(x, y, t, s), \phi(x)\right\rangle \rightarrow\langle\mu(x) G(x, y, t-s), \phi(x)\rangle
$$

in $\mathrm{U}_{\sigma, \nu}$ as $\mathrm{N} \rightarrow \infty$.

$$
\text { Hence } \quad \begin{aligned}
\lim _{\mathrm{N} \rightarrow \infty} \sum_{\mathrm{n}=0}^{N} a_{n} k_{n}\left\langle\mu(x) P_{n, \nu}(x,-s), \phi(x)\right\rangle \\
=\langle f(y),\langle\mu(x) G(x, y, t-s), \phi(x)\rangle\rangle .
\end{aligned}
$$

To complete the proof we have to show that 


$$
\lim _{s \rightarrow t-}\langle\mu(x) G(x, y, t-s), \phi(x)\rangle=\phi(y) \quad \text { in } U_{\sigma, \nu} \text {. }
$$

In other words, we need to show that for all $\mathrm{k}$ and $\varepsilon$, there exists $\delta(\mathrm{n}, \varepsilon)$ such that, for $0<t-s<\delta(n, \varepsilon)$,

$$
\exp \left(\mathrm{y}^{2} / 4 \sigma\right)\left|\int_{0}^{\infty} \Delta_{\mathrm{y}}^{\mathrm{k}}\{\mathrm{G}(\mathrm{x}, \mathrm{y}, \mathrm{t}-\mathrm{s})\} \phi(\mathrm{x}) \mathrm{d} \mu(\mathrm{x})-\phi_{\mathrm{k}}(\mathrm{y})\right|<\varepsilon .
$$

Since $\phi(y) \in U_{\sigma, \nu}$, we have for $y>A(n)$,

$$
\exp \left(\mathrm{y}^{2} / 4 \sigma\right)\left|\phi_{\mathrm{k}}(\mathrm{y})\right|<\frac{\varepsilon}{2}
$$

From Lemma 4.3 we know that there exists $A_{1}>A$ such that for $y>A_{1}(n)$ and $t<t_{0}\left(A_{1}(n)\right.$ independent of $\left.t\right)$,

$$
\exp \left(\mathrm{y}^{2} / 4 \sigma\right)\left|\int_{0}^{\infty} \Delta_{\mathrm{y}}^{\mathrm{k}}\{\mathrm{G}(\mathrm{x}, \mathrm{y}, \mathrm{t}-\mathrm{s})\} \phi(\mathrm{x}) \mathrm{d} \mu(\mathrm{x})\right|<\frac{\varepsilon}{2} .
$$

An application of Lemma 4.4 completes the proof of the theorem.

5. THE TEST FUNCTION SPACE $\mathrm{V}_{\sigma, \nu}$ (I) AND ITS DUAL.

Suppose $\Omega_{x, t}$ denotes the differential operator given by

$$
\Omega_{x, t} \equiv 4 t\left[\frac{d^{2}}{d x^{2}}-\frac{x^{2}}{16 t^{2}}+v(v-1) \frac{1}{x^{2}}\right],
$$

where $x \in I=(0, \infty)$ and $t$ is a fixed real number.

A complex-valued smooth function $\phi(x)$ belong to the space $v_{\sigma, \nu}$ (I) if

$$
\beta_{k}(\phi) \triangleq\left[\int_{0}^{\infty}\left|\Omega_{x, t}^{k} \phi(x)\right|^{2} d x\right]^{\frac{1}{2}}<\infty,
$$

where

$$
\mathrm{k}=0,1,2, \ldots \text {; and for each } \mathrm{n}, \mathrm{k}
$$

$$
\left(\Omega_{\phi, \psi_{n}}\right)=\left(\phi, \Omega^{k} \psi_{n}\right)
$$

Then $v_{\sigma, \nu}(I)$ is a linear space, and $\left\{\beta_{k}\right\}_{k=0}^{\infty}$ is a multinorm on $v_{\sigma, \nu}$. The topology over $\mathrm{V}_{\sigma, \nu}(\mathrm{I})$ is a subspace of $\mathrm{L}_{2}(\mathrm{I})$ when we identify each function in $\mathrm{V}_{\sigma, \nu}$ (I) with the corresponding equivalence class in $\mathrm{L}_{2}(\mathrm{I})$. Also convergence in $\mathrm{V}_{\sigma, \nu}(I)$ implies convergence in $\mathrm{L}_{2}(\mathrm{I})$. The space $\mathrm{V}_{\sigma, \nu}(\mathrm{I})$ is complete and therefore is Frechet . The dual space of $V_{\sigma, \nu}(I)$ is denoted by $V_{\sigma, \nu}^{\prime}(I)$. It can also be shown that $V_{\sigma, \nu}^{\prime}$ (I) is sequentially complete. 
We note that

$$
\Omega_{x, t} \psi_{n}(x, t)=-\lambda_{n} \psi_{n}(x, t)
$$

where

$$
\psi_{n}(x, t)=\left\{\frac{2^{\frac{1}{2}-\nu}}{\Gamma\left(\nu+\frac{1}{2}\right)}\right\}^{\frac{1}{2}} x^{\nu}[G(x, t)]^{\frac{1}{2}} \quad P_{n, \nu}(x,-t)
$$

with

$$
\lambda_{\mathrm{n}}=4 \mathrm{n}+2 \nu+1
$$

Therefore $\psi_{n}(x, t)$, as a function of $x$, is a member of $v_{\sigma, \nu}$ (I) for fixed $t$.

Following are important properties of these spaces: (i) for fixed $t, \Omega_{x, t}$ is a continuous linear mapping of $\mathrm{V}_{\sigma, \nu}$ (I) into itself. In view of the self adjoint nature of the operator $\Omega_{x, t}[$ see (5.1)] we define the generalized differential operator $\Omega_{x, t}$ on $V^{\prime}, \nu$ by

$$
\left(\Omega_{x, t} f(x), \phi(x)\right) \triangleq\left(f(x), \Omega_{x, t} \phi(x)\right),
$$

for fixed $t$, where $\mathrm{f} \in \mathrm{V}^{\prime}{ }_{\sigma, \nu}$ and $\phi \in \mathrm{v}_{\sigma, \nu}$.

(ii) $D(I)$ is a subspace of $V_{\sigma, \nu}(I)$, and the convergence in $D(I)$ implies convergence in $\mathrm{V}_{\sigma, \nu}(\mathrm{I})$. Consequently, restriction of any $\mathrm{f} \in \mathrm{V}_{\sigma, \nu}^{\prime}(I)$ to $D(I)$ is in $D^{\prime}$ (I). Moreover, convergence in $\mathrm{V}_{\sigma, \nu}^{\prime}(\mathrm{I})$ implies convergence in $D^{\prime}(\mathrm{I})$. (iii) $v_{\sigma, \nu}(I) \subset \varepsilon(I)$ and since $D(I)$ is dense in $\mathcal{E}(I), v_{\sigma, \nu}$ (I) is also dense in $\mathcal{E}(\mathrm{I})$. Furthermore, if $\left\{\phi_{\mathrm{m}}\right\}_{\mathrm{m}=1}^{\infty}$ converges in $\mathrm{V}_{\sigma, \nu}$ (I) to the limit $\phi$, then $\left\{\phi_{\mathrm{m}}\right\}$ also converges in $\mathcal{E}(I)$ to the some limit $\phi$. The space $\mathcal{E}^{\prime}(I)$ is a subspace of $V_{\sigma, \nu}^{\prime}$ (I). (iv) We imbed $\mathrm{L}_{2}$ (I) (and therefore, $V_{\sigma, \nu}$ (I) since $V_{\sigma, \nu}$ (I) $\subset \mathrm{L}_{2}$ (I) into $\mathrm{V}_{\sigma, \nu}^{\prime}$ (I) by defining the number that $f \in L_{2}$ (I) assigns to any $\phi, \in v_{\sigma, \nu}$ (I) as

$$
(f, \phi) \triangleq \int_{0}^{\infty} f(x) \overline{\phi(x)} d x .
$$

Then $\mathrm{f}$ is linear and continuous on $\mathrm{V}_{\sigma, \nu^{*}}$. This imbedding of $\mathrm{L}_{2}$ (I) into $\mathrm{V}_{\sigma, \nu}^{\prime}$ (I) is one to one.

(v) If $f(x)=\Omega_{x, t}^{k} g(x)$, for fixed $t$ and $g \in_{L_{2}}(I)$ and some $k$, then $f \in v_{\sigma, \nu}^{\prime}$ (I). Instead of working with the number $\langle\mathrm{f}, \phi\rangle$ that $\mathrm{f} \in \mathrm{V}_{\sigma, \nu}^{\prime}$ assigns to $\phi \in \mathrm{V}_{\sigma, \nu}$ it is more convenient to work with the number that $f$ assigns to the complex conjugate of $\phi$. We write 


$$
(f, \phi)=\langle f, \bar{\phi}\rangle \text {. }
$$

This is consistent with the inner product notation of $\mathrm{L}_{2}$ (I). The multiplication by a complex number a is given by

$$
(a f, \phi)=\langle a f, \bar{\phi}\rangle=\langle f, a \bar{\phi}\rangle=a(f, \phi) \text {. }
$$

The following two lemmas are useful in the subsequent analysis.

LEMMA 5.1. If $\phi(x) \in v_{\sigma, \nu}$ (I) then for $0<t \leq \sigma$,

$$
\phi(x)=\sum_{n=0}^{\infty}\left(\phi(x), \psi_{n}(x, t)\right) \psi_{n}(x, t),
$$

where the series converges in $\mathrm{v}_{\sigma, \nu}(I)$.

PROOF. By (5.2), $\Omega_{x, t}^{k} \phi(x)$ is in $L_{2}(I)$ for each nonnegative integer $k$. Hence by [5, pp. 470-471] we may expand $\Omega_{x, t}^{k} \phi(x)$ into a series of orthonormal functions $\psi_{n}(x, t)$. Thus for fixed $t, 0<t \leq \sigma$,

$$
\begin{aligned}
\Omega_{x, t}^{k}[\phi(x)] & =\sum_{n=0}^{\infty}\left(\Omega_{x, t}^{k} \phi(x), \psi_{n}(x, t) \psi_{n}(x, t)\right. \\
& =\sum_{n}\left(\phi(x), \Omega_{x, t}^{k} \psi_{n}(x, t)\right) \psi_{n}(x, t) \text { by } \\
& =\sum_{n}\left(\phi(x),\left(-\lambda_{n}\right)^{k} \psi_{n}(x, t)\right) \\
& =\sum_{n}\left(\phi(x), \psi_{n}(x, t)\right)\left(-\lambda_{n}\right)^{k} \psi_{n}(x, t) \\
& =\sum_{n=0}^{\infty}\left(\phi, \psi_{n}\right) \Omega_{x, t}^{k} \psi_{n} .
\end{aligned}
$$

Consequently, for each $k$,

$$
\beta_{k}\left[\phi(x)-\sum_{n=0}^{N}\left(\phi, \psi_{n}\right) \psi_{n}\right] \rightarrow 0 \text {, as } N \rightarrow \infty
$$

This proves the lemma.

Using (5.3), (5.12) and the fact that the inner product is continuous with repect to each of its arguments, for any two members $\phi$ and $x$ of $v_{\sigma, \nu}$, we obtain

$$
\left(\Omega_{x, t} \phi(x), \chi(x)\right)=\left(\phi(x), \Omega_{x, t} \chi(x)\right),
$$

Therefore the operator $\Omega_{x, t}$ is a self adjoint operator.

LEMMA 5.2. Let $a_{n}$ denote complex numbers. Then $\sum_{n=0}^{\infty} a_{n} \psi_{n}(x, t)$ for $0<t \leq \sigma$, converges in $v_{\sigma, \nu}$ (I) if and only if $\sum_{n=0}^{\infty}\left|\lambda_{n}\right|^{2 k}\left|a_{n}\right|^{2} \begin{aligned} & n=0 \\ & \text { converges for every non- }\end{aligned}$ negative integer $k$.

PROOF. The proof is similar to that of Zemaniar ¿ $\downarrow, \mathrm{p} 255]$ and hence it is omitted. 


\section{ORTHOGONAL SERIES EXPANSION OF GENERALIZED FUNCTIONS.}

The following theorem provides an orthonormal series expansion of generalized functions belonging to $\mathrm{V}_{\sigma, \nu}^{\prime}$ which in turn yields an inversion formula for a certain generalized integral transformation.

THEOREM 6.1. Let $F(n)$ be the generalized integral transformation of $f \in V_{\sigma, \nu}^{\prime}$ defined for fixed $t$ by

$$
F(n) \triangleq\left(f(x), \psi_{n}(x, t)\right) \equiv T[f]
$$

Then

$$
f(x)=\sum_{n=0}^{\infty} F(n) \psi_{n}(x, t)
$$

where the series converges in $V_{\sigma, \nu}^{\prime}(I)$.

PROOF. By Lemma 5.1, for any $\phi(x), \mathrm{V}_{\sigma, \nu}(I)$, we obtain

$$
\begin{aligned}
(f, \phi) & =\left(f, \sum\left(\phi(x), \psi_{n}(x, t)\right) \psi_{n}(x, t)\right) \\
& \left.=\sum_{n=0}^{\infty}\left(f, \psi_{n}\right) \overline{\left(\phi, \psi_{n}\right.}\right) \\
& =\sum_{n=0}^{\infty}\left(f, \psi_{n}\right)\left(\psi_{n}, \phi\right)=\sum_{n=0}^{\infty} F_{n}\left(\psi_{n}(x, t), \phi(x, t)\right) .
\end{aligned}
$$

This proves that the series on the right of $(6.1)$ converges in $V_{\sigma, \nu}^{\prime}(I)$.

THEOREM 6.2 (UNIQUENESS). Let $\mathrm{f}$ and $\mathrm{g}$ be elements of $\mathrm{V}_{\sigma, v}^{\prime}$ and let their transforms $F(n)$ and $G(n)$ satisfy $F(n)=G(n)$ for all $n$, then $f=g$ in the sense of equality in $\mathrm{V}_{\sigma, \nu}^{\prime}$.

PROOF. We have

$$
f-g=\sum\left(f-g, \psi_{n}\right) \psi_{n}=\sum\left[\left(f, \psi_{n}\right)-\left(g, \psi_{n}\right)\right] \psi_{n}=0 .
$$

7. CHARACTERIZATION THEOREMS. The following theorem gives a characterization of the transform $\mathrm{F}(\mathrm{n})$ of $\mathrm{f} \in \mathrm{V}_{\sigma, \nu}^{\prime}$. Its proof being similar to that of Theorem 9.6-1, p 261 in [1] and hence it is omitted.

THEOREM 7.1. Let $b_{n}$ denote complex numbers. Then for fixed, $t, o<t \leq \sigma$,

$$
\sum_{n=0}^{\infty} b_{n} \psi_{n}(x, t),
$$

converges in $\mathrm{V}_{\sigma, \nu}^{\prime}(\mathrm{I})$ if and only if there exists a nonnegative integer $\mathrm{q}$ such that the series 


$$
\sum_{\lambda \neq 0}\left|\lambda_{n}\right|^{-2 q}\left|b_{n}\right|^{2}
$$

converges. Furthermore, if $f$ denotes the sum in $v_{\sigma, \nu}^{\prime}$ of $(7.1)$ then $b_{n}=\left(f, \psi_{n}\right)$.

The next theorem is analogous to that of Theorem 9.6-2, p 262 [1] and gives a characterization of elements of $V_{\sigma, \nu}^{\prime}(I)$.

THEOREM 7.2. A necessary and sufficient condition for $f$ to be a member of $V_{\sigma, \nu}^{\prime}(I)$ is that there be some nonnegative integer $q$ and a $g \in L_{2}(I)$ such that for fixed $t$,

$$
f(x)=\Omega_{x, t}^{q} g(x)+\sum_{n=0} d_{n} \psi_{n}(x, t), o<t \leq \sigma,
$$

where $c_{n}$ are certain complex constants and $\sum_{n=0}$. denotes a summation on those $n$ for which $\lambda_{\mathrm{n}}=0$; these are finite in number.

\section{AN OPERATIONAL CALCULUS.}

Since $\Omega_{x, t}$ is a continuous linear mapping of $v_{\sigma, \nu}^{\prime}$ for every $f \in v_{\sigma, \nu}^{\prime}$ we can write

$$
\begin{aligned}
\Omega_{x, t}^{k} f(x) & =\sum_{n=0}^{\infty}\left(f(x), \psi_{n}(x, t)\right) \Omega_{x, t}^{k} \psi_{n}(x, t) \\
& =\sum_{n=0}^{\infty}\left(f(x), \psi_{n}(x, t)\right)\left(-\lambda_{n}\right)^{k} \psi_{n}(x, t)
\end{aligned}
$$

Using this fact we can solve the differential equation

$$
P\left(\Omega_{x, t}\right) u(x)=g(x),
$$

where $P$ is a polynomial and the given $g$ and unknown $u$ are required to be in $V_{\sigma, \nu}^{\prime}$ Applying the transformation $\mathrm{T}$ defined by (6.1) we obtain

$$
P\left(\lambda_{n}\right) U(n)=G(n), \quad U=T u, \quad G=T g .
$$

If $P\left(\lambda_{n}\right) \neq 0$ for every $n$, we can divide $P\left(\lambda_{n}\right)$ and apply $T^{-1}$ to obtain

$$
u=\sum_{n=0}^{\infty} \frac{G(n)}{P\left(\lambda_{n}\right)} \psi_{n} \text {. }
$$

By Theorems 6.2 and 7.1 , this solution exists, and is unique in $V_{\sigma, \nu}^{\prime}$. If $P\left(\lambda_{n}\right)=0$ for some $\lambda_{n}$, say, for $\lambda_{n_{k}}(k=1, \ldots, m)$, then a solution exists in $v_{\sigma, v}^{\prime}$ if and only if $G\left(\lambda_{n_{k}}\right)=0$ for $k=1,2, \ldots, m$. In this case solution (8.3) is no longer unique and we may add to it any complementary solution

$$
u_{c}=\sum_{k=1}^{m} a_{k} \psi_{n_{k}} \text {, where } a_{k} \text { are arbitrary constants, }
$$




\section{REFERENCES}

1. ZEMANIAN, A.H. Generalized Integral Transformation, Interscience, New York (1968).

2. KOREVAAR, J. Pansions and the Theory of Fourier Transforms, Trans. Amer. Math. Soc. 91 (1959), 53-101.

3. GELFAND, I.M. and SILOV, G.E. Fourler Transforms of Rapidly Increasing Functions and Questions of Uniqueness of the Solution of Cauchy's Problem, Uspehi. Mat. Nauk. (N.S.) Vol. 8 (58) (1963) pp. 3-54.

4. EHRENPREIS, L. Analytic Functions and the Fourier Transform of Distributions I, Ann. Math. Vo1. 63 (1956) pp. 129-159.

5. HATMO, D.T. $\mathrm{L}^{2}$ expansions in Terms of Generalized Heat Polynomials and of their Appell Transforms, Pacific J. Math. 15 (1965), pp. 865-875.

6. HAIMO, D.T. Expansions in Terms of Generalized Heat Polynomials and of their Appe11 Transforms, J. Math. Mech. 15 (1966), 735-758.

7. ROSENBLOOM, P.C. \& WIDDER, D.V. Expansions in Terms of Heat Polynomials and Associated Functions, Trans. Amer. Math. Soc. 92 (1959), 220-266.

8. ERDELYI, A. Higher Transcendental Functions, Vol. II, McGraw-Hi11, New York (1953).

9. SCHWARTZ, L. Theorie des Distributions, Tomes I, II, Hermann Paris 1950, 1951.

10. PANDEY, J.N. Complex Inversion for the Generalized Hankel Convolution Transformation, SIAM J. App1. Math. 17 (5) (1969), pp. 835-848.

11. PANDEY, J.N. The Generalized Welerstrass Hankel Convolution Transform, SIAM J. App1. Math. 20 (1) (1971), pp. 110-123. 


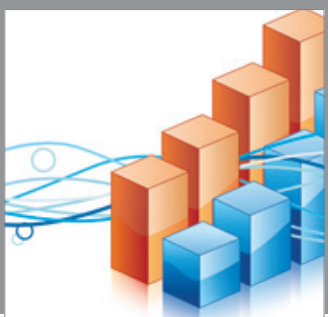

Advances in

Operations Research

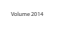

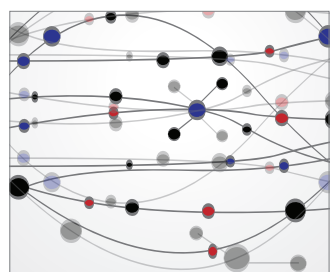

\section{The Scientific} World Journal
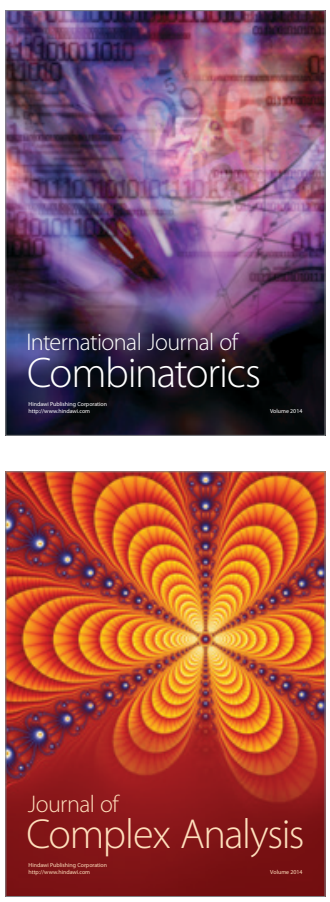

International Journal of

Mathematics and

Mathematical

Sciences
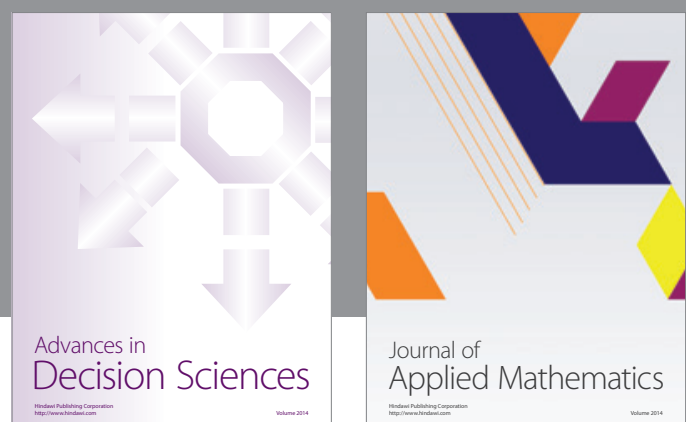

Journal of

Applied Mathematics
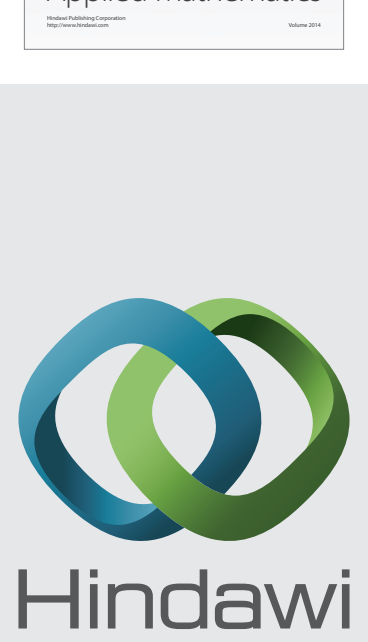

Submit your manuscripts at http://www.hindawi.com
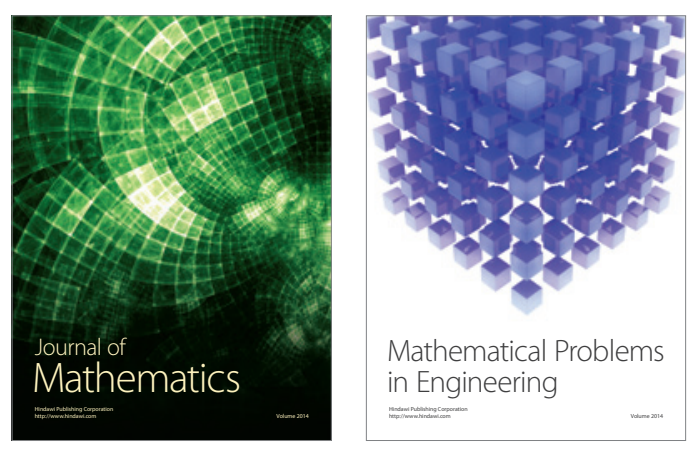

Mathematical Problems in Engineering
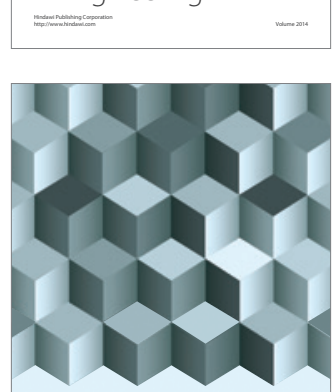

Journal of

Function Spaces
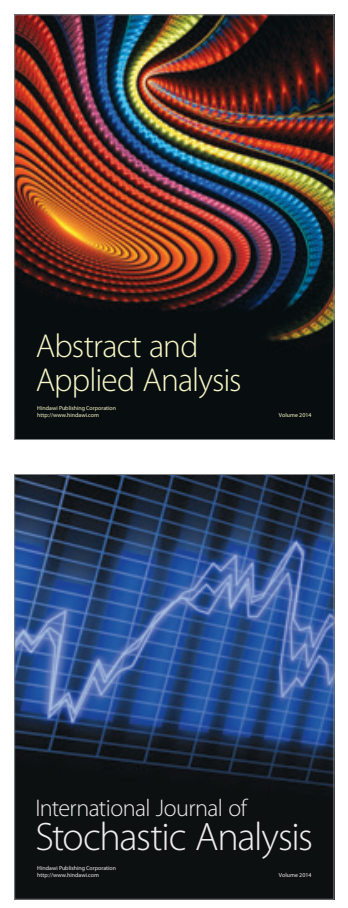

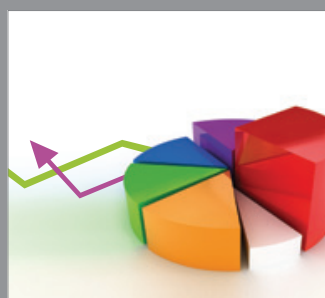

ournal of

Probability and Statistics

Promensencen
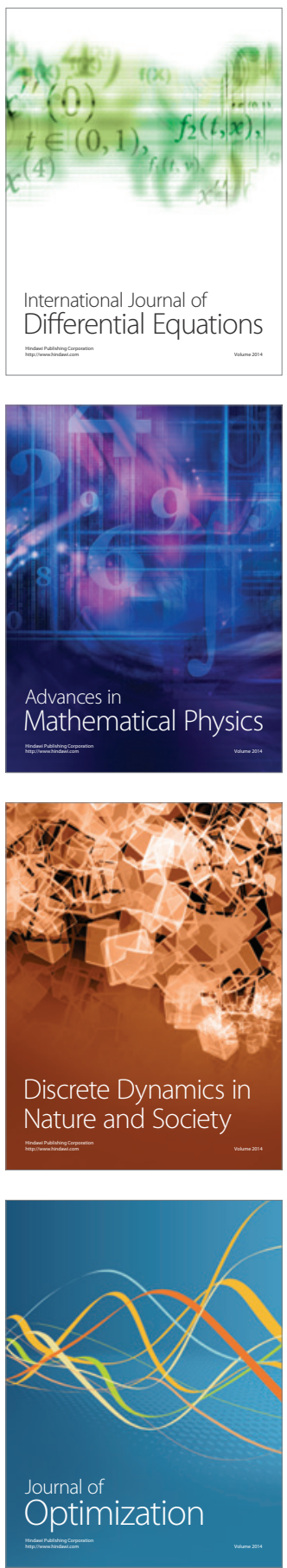